\title{
X-ray Transient Sources (Multifrequency Laboratories) The Case of the Prototype A0535+26/HDE 245770
}

\author{
F. Giovannelli, L. Sabau-Graziati
}

\begin{abstract}
The goal of this paper is to discuss the behaviour of the X-ray transient source A0535+26 which is considered for historical reasons and for the huge amount of multifrequency data, spread over a period of 35 years, as the prototype of this class of objects. Transient sources are formed by a Be star - the primary — and a neutron star X-ray pulsar — the secondary - and constitute a sub-class of X-ray binary systems.

We will emphasize the discovery of low-energy indicators of high-energy processes. They are UBVRI magnitudes and Balmer lines of the optical companion. Particular unusual activity of the primary star - usually at the periastron passage of the neutron star - indicates that an X-ray flare is drawing near. The shape and intensity of X-ray outbursts are dependent on the strength of the activity of the primary.

We derive the optical orbital period of the system as $110.856 \pm 0.02$ days. By using the optical flare of December 5 , 1981 (here after 811205-E) that triggered the subsequent X-ray outburst of December 13, 1981, we derive the ephemeris of the system as $\mathrm{JD} \mathrm{P}_{\mathrm{opt}-\text { outb }}=\mathrm{JD}_{0}(2,444,944) \pm n(110.856 \pm 0.02)$. Thus the passage of the neutron star at the periastron occurs with a periodicity of $110.856 \pm 0.02$ days and the different kinds of X-ray outbursts of A0535+26 following the definitions reported in the review by Giovannelli \& Sabau-Graziati (1992) — occur just after $\sim 8$ days. The delay between optical and X-ray outbursts is just the transit time of the material coming out from the optical companion to reach the neutron star X-ray pulsar.

The occurrence of X-ray "normal outbursts", "anomalous outbursts" or "casual outbursts" is dependent on the activity of the Be star: "quiet state: steady stellar wind", "excited state: stellar wind plus puffs of material", and "expulsion of a shell", respectively. In the latter case, the primary manifests a strong optical activity and the consequent strong X-ray outburst can occur in any orbital phase, with a preference at the periastron passage of the neutron star, because of its gravitational effects on the Be star.
\end{abstract}

Keywords: X-ray/Be systems, X-ray pulsars, be stars, optical, spectroscopy, photometry, X-rays, individual: A0535 +26 $\equiv 1 \mathrm{~A} 0535+26 \equiv 4 \mathrm{U} 0538+26 \equiv 1 \mathrm{H} 0536+263 \equiv 1 \mathrm{RXS}$ J053855.1+261843, individual: HDE $245770 \equiv \mathrm{BD}^{2} 26^{\circ} 883 \equiv$ $\mathrm{V} 725 \mathrm{Tau} \equiv$ AAVSO $0532+26 \equiv \mathrm{SAO} 77348$.

\section{Introduction}

The trivial definition of X-ray binaries (XRBs) is that they are binary systems emitting X-rays. However it has been largely demonstrated that X-ray binary systems emit energy in IR, Optical, UV, X-ray, Gammaray energy ranges, and they sometimes also show valuable radio emission. They can be divided into different sub-classes

- High Mass X-ray Binaries (HMXB), in which the optical companion is an early type giant or supergiant star and the collapsed object is a neutron star or a black hole. They are concentrated around the galactic plane. The mass transfer usually occurrs via stellar wind; they show hard pulsed X-ray emission (from 0.069 to $1413 \mathrm{~s}$ ) with $\mathrm{KT} \geq 9 \mathrm{keV}$; typical X-ray luminosity ranges from $10^{34}$ to $10^{39} \mathrm{erg} \mathrm{s}^{-1}$, and the ratio of X-ray to optical luminosity is $\sim 10^{-3}-10$. HMXBs can be divided into two sub-classes

- Hard Transient X-ray Sources (HXTS) in which the neutron star is eccentri- cally $(\mathrm{e} \sim 0.2-0.5)$ orbiting around a V-III luminosity-class $\mathrm{Be}$ star $\left(\mathrm{P}_{\text {orb }}>10\right.$ days $)$; they show strong variable pulsed hard $\mathrm{X}$ ray emission $\left(\mathrm{L}_{\mathrm{Xmax}} / \mathrm{L}_{\mathrm{Xmin}}>100\right)$ with $\mathrm{KT} \geq 17 \mathrm{keV}$;

- Permanent X-ray Sources (PXS), in which the neutron star or black hole is circularly orbiting $(\mathrm{e} \sim 0)$ around a giant or supergiant $\mathrm{OB}$ star $\left(\mathrm{P}_{\text {orb }}<10\right.$ days $)$; they show an almost steady permanent pulsed hard Xray emission $\left(\mathrm{L}_{\mathrm{Xmax}} / \mathrm{L}_{\mathrm{Xmin}} \ll 100\right)$;

- Obscured Sources, which display a huge amount of low energy absorption produced by the dense wind of the supergiant companion;

- Supergiant Fast X-ray transients (SFXT), a new subclass of transients in which the formation of transient accretion discs could be partly responsible for the flaring activity in systems with narrow orbits. 
- Low Mass X-ray Binaries (LMXB), in which the optical companion is a low-mass-late-type star and the collapsed object is a neutron star or a black hole ( $\mathrm{P}_{\text {orb }}$ from 41 min to 11.2 days). They are concentrated around the galactic plane and especially in the galactic center. The mass transfer in these systems usually occurs via Roche lobe overflow. Their emission in the soft X-ray range is usually not pulsed with $\mathrm{KT} \leq 9 \mathrm{keV}$. Their X-ray luminosity ranges from $10^{36}$ to $10^{39}$ erg s${ }^{-1}$ and $\mathrm{L}_{\mathrm{X}} /$ Lopt $\sim 10^{2}-10^{4}$; many LMXBs show Quasi Periodic Oscillations (QPOs) between 0.02 and 1000 seconds, and a few of them also show pulsed X-ray emission, such as Her X1, $4 \mathrm{U}$ 1626-27 and GX 1+4.

- Cataclysmic Variables (CVs), in which the optical companion is a low-mass-late-type star and the compact object is a white dwarf. The detected CVs are spread roughly around the solar system at a distance of 200-300 pc. The orbital periods range from tens of minutes to about ten hours. The mass transfer occurs either via Roche lobe overflow or via accretion columns or in an intermediate way, depending on the value of the magnetic field. Typical X-ray luminosity ranges from $10^{32}$ to $10^{34} \mathrm{erg} \mathrm{s}^{-1}$. An updated review on CVs is that of Giovannelli (2008);

- RS Canum Venaticorum (RS CVn) type systems, in which no compact objects are present and the two components are an $\mathrm{F}$ or $\mathrm{G}$ hotter star and a $\mathrm{K}$ star. Typical X-ray luminosity ranges from $10^{30}$ to $10^{31} \mathrm{erg} \mathrm{s}^{-1}$. In the current literature they are usually excluded from the class of X-ray binaries, since historically they were discovered as X-ray emitters only with the second generation of X-ray experiments.

For a general review of XRBs, see the paper by Giovannelli \& Sabau-Graziati (2004). XRBs are the best laboratory for the study of accreting processes thanks to their relatively high luminosity in a large part of the electromagnetic spectrum. For this reason, multifrequency observations are fundamental in understanding their morphology and the physics governing their behaviour. Because of the strong interactions between the optical companion and the collapsed object, low and high energy processes are strictly related. It is often easier to perform observations of low energy processes (e.g. in the radio, near-infrared (NIR) and optical bands), since the experiments are typically ground-based, unlike observations of high energy processes, for which experiments are typically space-based.

$\mathrm{X}$-ray/Be binaries are the most abundant group of massive X-ray binaries in the galaxy, with a total inferred number of between $10^{3}$ and $10^{4}$. Those which occasionally flare-up as transient X-ray/Be systems are only the "tip" of this vaste "iceberg" of systems (van den Heuvel and Rappaport, 1987). The mass loss processes are due to the rapid rotation of the Be star, the stellar wind and, sporadically, to the expulsion of a casual quantity of matter essentially triggered by gravitational effects close to the periastron passage of the neutron star. The long orbital period ( $>10$ days) and the large eccentricity of the orbit $(>0.2)$ together with the transient hard X-ray behavior are the main characteristics of these systems. Among the whole sample of galactic systems containing $114 \mathrm{X}$-ray pulsars (Johnstone, 2005), only few have been extensively studied. Among these, the system A $0535+26 / \mathrm{HDE} 245770$ is the best known, thanks to concomitant favorable causes, which have enabled thirty five years of coordinated multifrequency observations, most of them discussed by e.g. Giovannelli \& Sabau-Graziati (1992), Burger et al. (1996), Piccioni et al. (1999). Accretion powered X-ray pulsars usually capture material from the optical companion via the stellar wind, since this primary star generally does not fill its Roche lobe. However, in some specific conditions (e.g. the passage at the periastron of the neutron star) and in particular systems (e.g. A $0535+26 / \mathrm{HDE} 245770$ ), the formation of a temporary accretion disk around the neutron star behind the shock front of the stellar wind is possible. This enhances the efficiency of the process of mass transfer from the primary star onto the secondary collapsed star, as discussed by Giovannelli \& Ziolkowski (1990) and by Giovannelli et al. (2007) in the case of A $0535+26$.

Optical emission of HMXBs is dominated by the emission of the optical primary component, which is not, in general, strongly influenced by the presence of the X-ray source. The behavior of the primary stars can be understood in the classical (or almost) framework of the astrophysics of these objects, i.e. by studying their spectra, which will provide indications on mass, radius, and luminosity. The two groups of HMXBs differ because of the different origin of the mass loss process: in the first group, the mass loss process occurs via a strong stellar wind and/or because of an incipient Roche lobe overflow; in the second group, the mass transfer is probably partially due to the rapid rotation of the primary star and partially due to a stellar wind and sporadically due to expulsions of a casual quantity of matter, essentially triggered by gravitational effects because of periastron passage, where the effect of the secondary collapsed star is more marked. A relationship between the orbital period of HMXBs and the spin period of X-ray pulsars is shown in Fig. 1 (updated from Giovannelli \& Sabau-Graziati, 2001 and from Corbet, 1984, 1986). We can recognize three kinds of systems, namely disk-fed, wind-fed $\left[\mathrm{P}_{\text {pulse }} \propto(P \text { orb })^{4 / 7}\right]$, and $\mathrm{X}$-ray/Be systems $\left[\mathrm{P}_{\text {pulse }} \propto(P \text { orb })^{2}\right]$. 


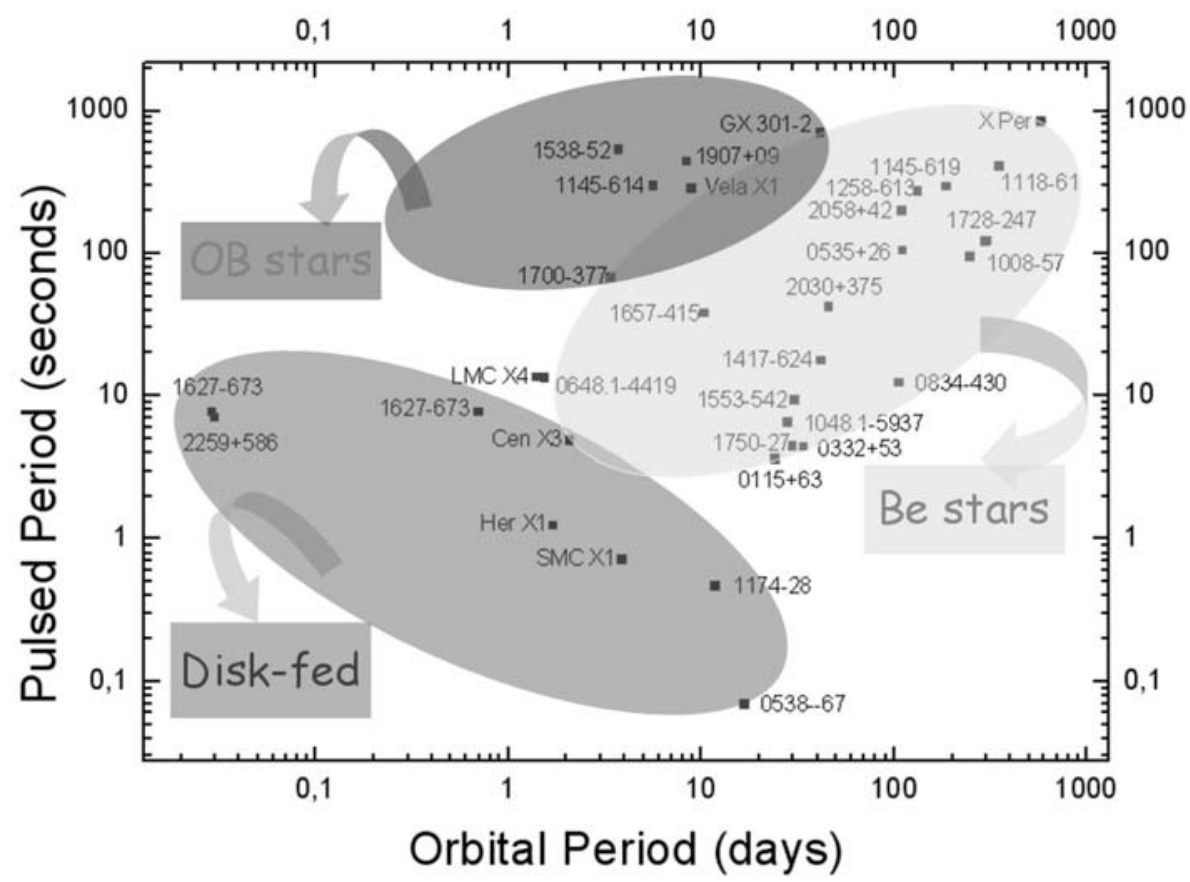

Fig. 1: Spin period vs orbital period for X-ray pulsars. Disk-fed systems are clearly separated by systems having as optical counterparts either OB stars or Be stars (Giovannelli \& Sabau-Graziati, 2001)

Most of the systems having a Be primary star are hard X-ray $(\mathrm{KT}>10 \mathrm{KeV})$ transient sources (HXTS). They are concentrated on the galactic plane within a band of $\sim 3.9^{\circ}$. The orbits are quite elliptic and the orbital periods are large (i.e. A 053866: $\mathrm{e}=0.7$, Porb $=16.6$ days (Skinner et al., 1982); A 0535+26: e = 0.47 (Finger, Wilson \& Hagedon, 1994), $\mathrm{P}=111.0$ days (Priedhorsky \& Terrell, 1983). The X-ray flux during the outburst phases is of the order 10-1000 times greater than during quiescent phases. For this reason, on the contrary, the stars belonging to the first class which do not present such strong variations in X-ray emission can be named "standard" high mass X-ray binaries. In Xray/Be systems, the primary Be star is relatively unevolved and is contained within its Roche lobe. The strong outbursts occur almost periodically in time scales of the order of weeks or months. Their duration is shorter than the quiescent phases. During Xray outbursts, spin-up phenomena in several systems have been observed (i.e. A $0535+26$ and $4 \mathrm{U} 1118-61$ (Rappaport \& Joss, 1981)). The observed spin-up rates during the outbursts are consistent with torsional accretion due to an accretion disk (e.g. Ghosh, 1994). The formation of a temporary accretion disk around the collapsed object should therefore be possible during the outburst phases (e.g. Giovannelli \& Ziolkowski, 1990).

The number of X-ray pulsars increases slowly with time, thanks to new detections performed at various new generation observatories. There were 95 (Giovannelli \& Sabau-Graziati, 2000) in 2000 and the orbital periods were known only for about three dozen of them. They contain the group of permanent HMXBs and the group of transient HMXBs (X-ray/Be systems), whose components are an X-ray pulsing neutron star — the secondary and a giant or supergiant $\mathrm{OB}$ or a Be star, respectively - the primary. Moreover, some lowmass X-ray Binaries (LMXBs) containing an Xray pulsar, and some pulsars belonging to Magellanic Clouds were also contained in the sample of 95 systems. In 2005, there were 114 known Xray pulsars (Johnston, 2005). $47 \mathrm{X}$-ray pulsars have been detected in the SMC (Corbet et al., http://lheawww.gsfc.nasa.gov/users/corbet/pulsars/). Recently, Rajoelimanana, Charles \& Udalski (2010) listed 49 optical counterparts of SMC X-ray pulsars detected by MACHO and OGLE. There are 20 systems with known $\mathrm{P}_{\text {spin }}$, while there are 23 systems with known $\mathrm{P}_{\text {orb }}, 6$ of which are uncertain.

In this paper we will discuss the history of the discovery of optical indicators of high energy emission in the prototype system A0535+26/HDE 245770, updated to the March-April 2010 event, when strong optical activity occurred roughly 8 days before the X-ray outburst (Caballero et al., 2010a) that was predicted by Giovannelli et al. (2010).

\section{The X-ray/Be System A0535+26/HDE 245770}

A $0535+26 /$ HDE 245770 is a typical X-ray/Be runaway system (van Oijen 1989), whose components are a $104 \mathrm{~s}$ X-ray pulsar (Rosenberg et al. 1975) and an O9.7 IIIe star (Giangrande et al. 1980). The X-ray 


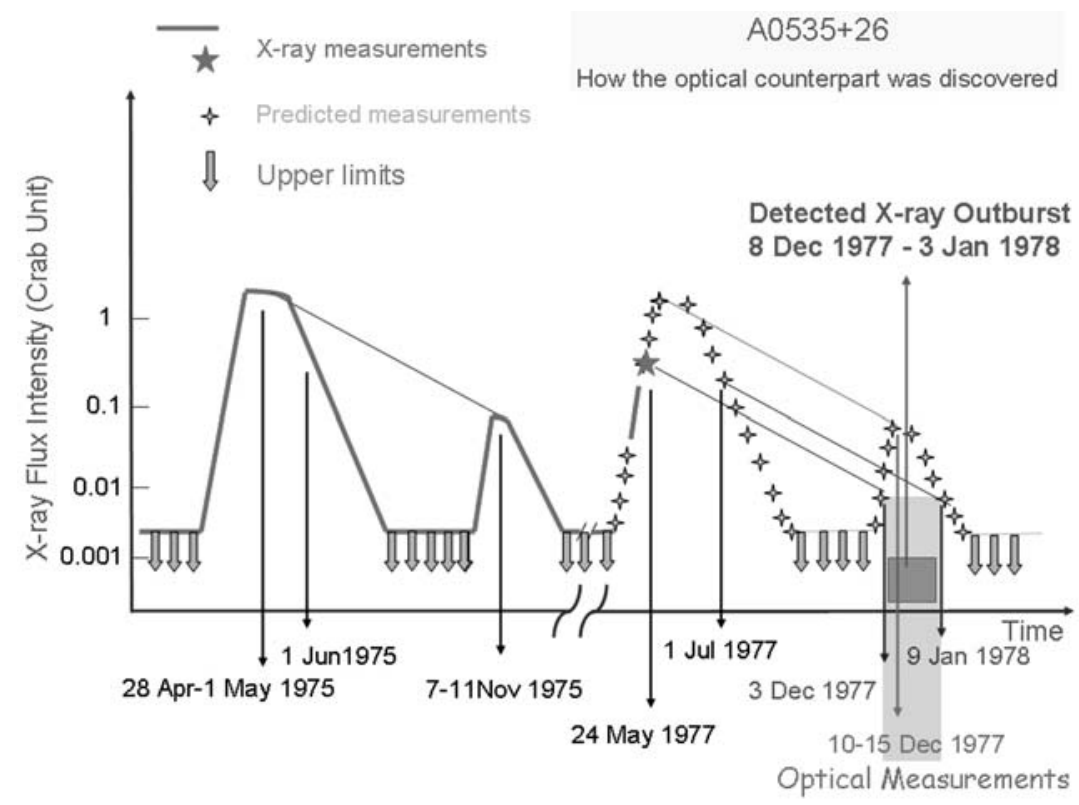

Fig. 2: X-ray flux versus time of A $0535+26$. X-ray measurements are reported with red lines and asterisks, upper limits with green arrows, and predicted fluxes with light blue stars. Periods of real detected X-ray outburst and optical measurements are also marked (Giovannelli, 2005)

pulsar is spinning up with a pulse period derivative $\dot{\mathrm{P}} / \mathrm{P}=-4.9 \times 10^{-4} \mathrm{yr}^{-1}$ (Coe et al. 1990), with alternating phases of spin-down during X-ray outbursts. The X-ray pulsar A0535+26 has a magnetic field of $\sim 10^{13} \mathrm{G}$ (Grove et al. 1995). The orbital period has been determined by many authors, using various methods: $111^{\mathrm{d}} .0 \pm 0^{\mathrm{d}} .4$ (Priedhorsky \& Terrell 1983), $111^{\mathrm{d}} .38 \pm 0^{\mathrm{d}} .11$ (Motch et al. 1991), and $110^{\mathrm{d}} .3 \pm 0^{\mathrm{d}} .3$ (Finger, Wilson \& Hagedon 1994) from X-ray data, and $110^{\mathrm{d}} .0 \pm 0^{\mathrm{d}} .5$ (Coe et al., 2006) from the increasing X-ray activity associated with the periastron passage of the neutron star. These values agree with the value reported by Guarnieri et al. (1985) from longterm optical photoelectric data $\left(109^{\mathrm{d}} .8 \pm 2^{\mathrm{d}} .0\right)$. The orbital eccentricity of the system is 0.47 (Finger, Wilson \& Hagedon 1994). The terminal velocity of the stellar wind from the star with significant optical output is $\sim 630 \mathrm{~km} \mathrm{~s}^{-1}$ (Giovannelli et al. 1982), and its mass loss rate is $\sim 10^{-8} \mathrm{M}_{\odot} \mathrm{yr}^{-1}$ (Giovannelli et al. 1984a; de Loore et al. 1984).

The X-ray pulsar is close to its equilibrium state (Bisnovatyi-Kogan 1991; Li \& van den Heuvel 1996), which could be a reason why some of the expected Xray outbursts have failed to occur. This was noted at the end of the 1980s, as discussed by Giovannelli \& Ziółkowski (1990, and references therein), or in the mid-nineties, as found by the RXTE satellite (see http://space.mit.edu/XTE). The O9.7 IIIe companion does not normally fill its Roche lobe (de Loore et al. 1984), although a temporary accretion disc can be formed around the neutron star when it is close to periastron (Giovannelli \& Ziółkowski 1990; Giovannelli et al., 2007).
Complete reviews of the A0535+26/HDE 245770 system can be found in the papers by Giovannelli et al. (1985), Giovannelli \& Sabau-Graziati (1992), and Burger et al. (1996).

\subsection{Historical Multifrequency Observations of A $0535+26 / \mathrm{HDE}$ 245770}

The most studied HMXB system, for historical reasons and due to concomitant favourable causes, is the X-ray/Be system A $0535+26 / \mathrm{HDE} 245770$. By means of long series of coordinated multifrequency measurements, very often simultaneously obtained, it was possible to:

- identify the optical counterpart HDE 245770 of the X-ray pulsar;

- identify various X-ray outbursts triggered by different states of the optical companion and influenced by the orbital parameters of the system;

- identify the presence of a temporary accretion disc around the neutron star at periastron.

Multifrequency observations of this system started soon after its discovery as an X-ray pulsar by the Ariel-5 satellite (Rosenberg et al., 1975). The error box of the X-ray source contains 11 stars up to 23rd magnitude; these include the 9 mag Be star HDE 245770. The a priori probability of finding a $9 \mathrm{mag}$ star in such a field is 0.004 , so that Margon et al. (1977) suggested this star as a probable optical counterpart of $\mathrm{A} 0535+26$. But in order to really associate this star with the X-ray pulsar, it was necessary to find a clear signature proving that the 
two objects would belong to the same binary system. This happened thanks to a sudden insight of one of us (FG), who predicted the fourth X-ray outburst of A $0535+26$ around mid December 1977. For this reason, Giovannelli's group was observing in optical HDE 245770 around the predicted period for the Xray outburst of A $0535+26$. Figure 2 shows the Xray flux intensity of $\mathrm{A} 0535+26$ as deduced by various measurements available at that time, with obvious meaning of the symbols used (Giovannelli, 2005). FG's intuition was sparked by looking at the rise of the X-ray flux (red line) and at the 24th May 1977 measurement (red asterisk): he assumed that the evident rise of the X-ray flux would have produced an outburst similar to the first one, which occurred in 1975. Then with a simple extrapolation he predicted the fourth outburst, similar to the second: and this happened!

Optical photoelectric photometry of HDE 245770 showed significant light enhancement of the star relative to the comparison star BD +26 876 between Dec 17 and Dec 21 (here after 771220-E) and successive fading up to Jan. 6 (Bartolini et al., 1978), whilst satellite SAS-3 was detecting an X-ray flare (Chartres \& Li, 1977). The observed enhancement of optical emission followed by the flare-up of the Xray source gave a direct argument strongly supporting the identification of HDE 245770 - later nicknamed Flavia' star by Giovannelli \& Sabau-Graziati (1992) — with A $0535+26$.

Soon after, with spectra taken at the Loiano 152 cm telescope with a Boller \& Chivens 26767 grating spectrograph (831 grooves/mm II-order grating: $39 \AA \mathrm{mm}^{-1}$ ) onto Kodak $103 \mathrm{aO}$ plates, it was possible to classify HDE 245770 as O9.7IIIe star. This classification was so good that it survives even to the recent dispute attempts made with modern technology. The mass and radius of the star are $15 \mathrm{M}_{\odot}$ and $14 \mathrm{R}_{\odot}$, respectively; the distance to the system is $1.8 \pm 0.6 \mathrm{kpc}$ (Giangrande et al., 1980).

UV spectra taken with the IUE enabled the reddening of the system to be determined as E(B$\mathrm{V})=0.75 \pm 0.05 \mathrm{mag}$, the rotational velocity of the O9.7IIIe star $\left(\mathrm{v}_{\text {rot }} \sin i=230 \pm 45 \mathrm{~km} \mathrm{~s}^{-1}\right)$, the terminal velocity of the stellar wind $\left(\mathrm{v}_{\infty} \simeq 630 \mathrm{~km} \mathrm{~s}^{-1}\right)$, the mass loss rate $\left(\dot{\mathrm{M}} \sim 10^{-8} \mathrm{M}_{\odot} \mathrm{yr}^{-1}\right.$ in quiescence (Giovannelli et al., 1982). During the October 1980 strong outburst, the mass loss rate was $\dot{\mathrm{M}} \sim 7.7 \times 10^{-7} \mathrm{M}_{\odot} \mathrm{yr}^{-1}$ (de Martino et al., 1989).

\subsection{Optical and Ultraviolet indicators of X-ray outbursts in $\mathrm{A} 0535+26 / \mathrm{HDE} 245770$}

Giovannelli et al. (1985) and Guarnieri et al. (1985) found UV and optical features of HDE 245770 as in- dicators of the X-ray activity of A $0535+26$. They found experimentally that the X-ray flaring activity of A $0535+26$ is preceded by modifications in the optical spectrum of the companion HDE 245770, of about one week $\left(\approx 0.6 \times 10^{6} \mathrm{~s}\right)$. This roughly corresponds to the transit time of puffs of material expelled by the Be star at $\sim 300 \mathrm{~km} \mathrm{~s}^{-1}$ in order to reach the neutron star, being the dimensions of the orbit of $\sim 1.34 \mathrm{AU}$ (de Loore et al., 1984). The $\mathrm{H}_{\gamma}$ line seems to be the best indicator of the activity going on. Moreover, enhancements of optical luminosity were observed on four occasions before X-ray outbursts (Bartolini et al., 1983; Maslennikov, 1986). Narrow absorption components present in the blue wings of the Si IV and C IV resonance lines indicate a variable mass loss superimposed on a steady wind of $\sim 10^{-8} \mathrm{M}_{\odot}$ yr-1 (Giovannelli et al., 1984a; de Loore et al., 1984). They are strong probes in studying the physics and dynamics of the mass transfer at periastron and the subsequent $\mathrm{X}$-ray flaring. Xray outbursts are triggered by the eccentric orbital motion $\left(\right.$ Porb $\cong 111$ days: $\approx 10^{7} \mathrm{~s}$ ). Then, multifrequency monitoring of this system and of a number of other X-ray/Be systems around the passage at the periastron could give a conclusive answer to many of the open problems on the Be/X-ray transients.

From two outburst decays with exactly the same shape, shown in Fig. 3 (Bartolini et al., 1983) it is possible to derive the optical period of the system $\left(\mathrm{P}_{\text {orb-opt }}=110.856 \pm 0.02\right.$ days. This value is in agreement with the many values available in the literature, but it has the advantage that it has been derived by two simple optical measurements. We will use such a period in the following in order to discuss the flaring activity of the system.

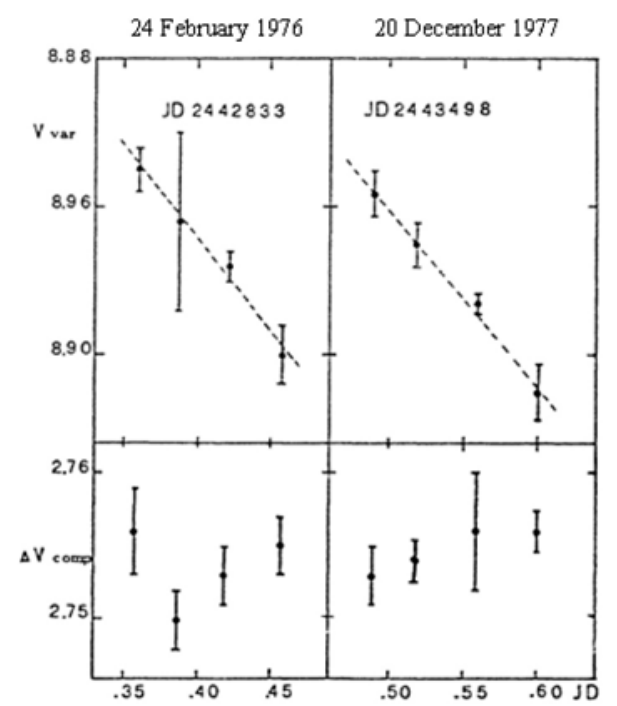

Fig. 3: Decay from two outbursts of optical luminosity of HDE 245770: the first on February 24, 1976, the second on December 20, 1977 (Bartolini et al., 1983). 


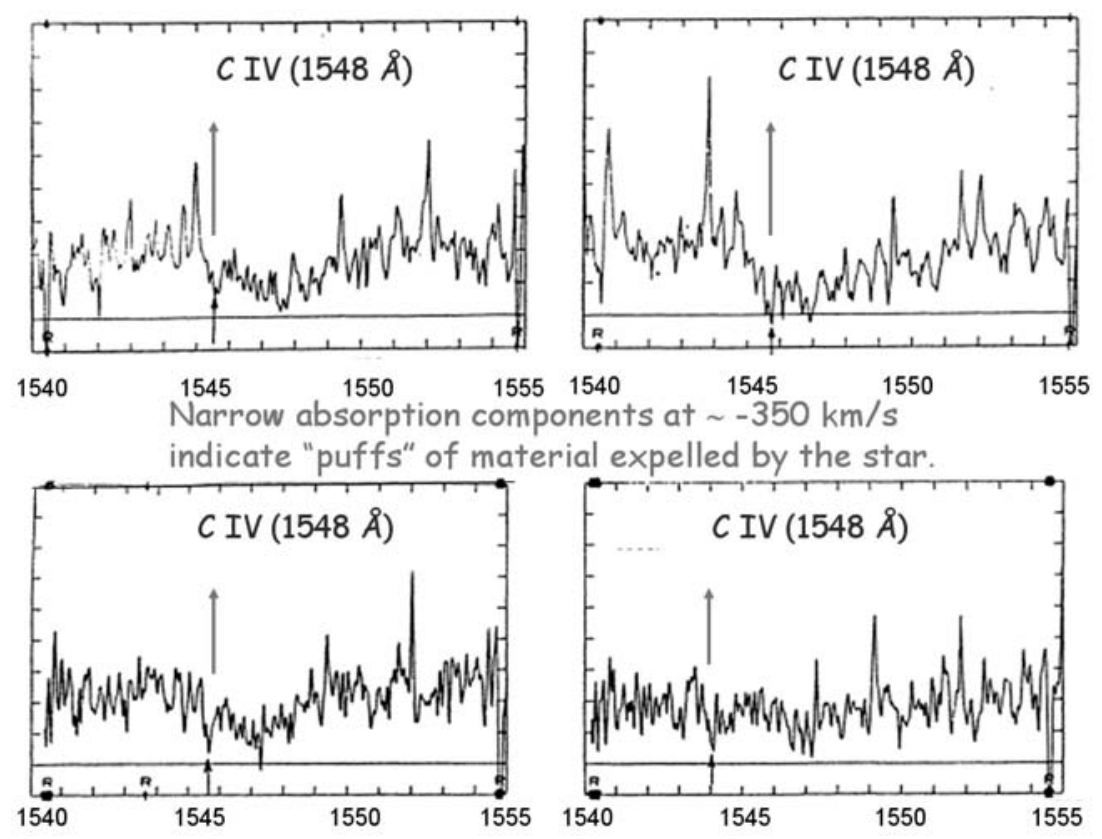

Fig. 4: C IV resonance line of Flavia' star with narrow absorption components marked with red arrows. The data comes from the IUE short wavelength high resolution spectra. Clockwise from the upper right panel, the spectra were taken by Giovannelli on November 1, 1981 (X-ray quiescence), October 26, 1980 (X-ray outburst decay), January 23, 1984 (X-ray outburst), and November 10, 1983 (X-ray quiescence), respectively
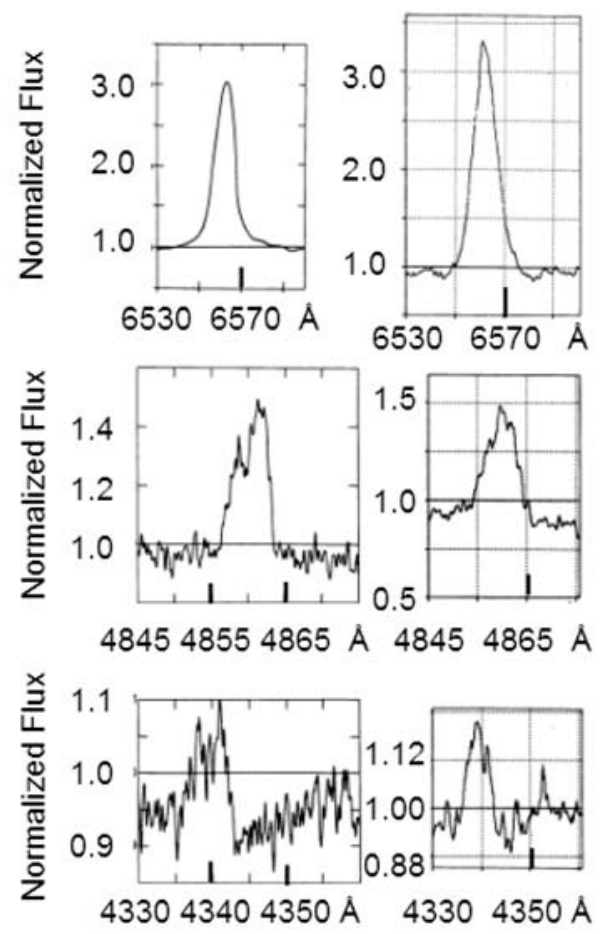

Fig. 5: Typical $\mathrm{H}_{\alpha}, \mathrm{H}_{\beta}$ and $\mathrm{H}_{\gamma}$ lines of Flavia' star before X-ray activity of the neutron star. The fluxes are normalized to the continuum. The left and right panels report spectra taken at Haute Provence and Loiano Observatories, respectively (Giovannelli et al., 1984a)

Figure 4 shows the narrow absorption components in the $\mathrm{C}$ IV resonance line in the high resolution IUE spectra of Flavia' star. The velocity of the "puffs" of material expelled by the star is $\sim 350 \pm 50 \mathrm{~km} \mathrm{~s}^{-1}$, roughly coincident with that measured in the optical spectra $\left(\sim 300 \pm 50 \mathrm{~km} \mathrm{~s}^{-1}\right)$. Then, the transit time from the primary to the secondary is about 7-8 days, being the dimension of the system of $~ 1.34 \mathrm{AU}$ (Giovannelli et al., 1984a; de Loore et al., 1984; Guarnieri et al., 1985b).

Figure 5 shows typical $\mathrm{H}_{\alpha}, \mathrm{H}_{\beta}$ and $\mathrm{H}_{\gamma}$ lines of Flavia' star before X-ray activity of the neutron star. The spectra were taken at the Haute Provence and Loiano Observatories (Giovannelli et al., 1984a).

Figure 6 shows the optical outburst 811205-E (Guarnieri et al., 1985b) - marked with a red line which occurred 8 days before the X-ray outburst of December 13, 1981 (Nagase et al., 1982) — marked with a blue line - and was just 13 cycles after 771220-E), when, through enhanced optical activity, Bartolini et al. (1978) discovered the firm association between the X-ray pulsar and the O9.7 IIIe star. Around the strong X-ray outburst - October 9-24, 1980: JD 2444522 - 2444537 - (marked with the blue rectangle in Fig. 6) scarce optical measurements are available. However, such a strong X-ray outburst occurred between the 9th (JD 2444500.6) and 10th (JD $2444611.4)$ cycle after $771220-\mathrm{E}$. This will be commented in the conclusions.

We use 811205 -E to give the optical ephemerides of the binary system that is in our opinion the most reliable. They are:

$$
\mathrm{JD}_{\text {opt-outb }}=\mathrm{JD}_{0}(2,444,944) \pm \mathrm{n}(110.856 \pm 0.02)
$$
days. 


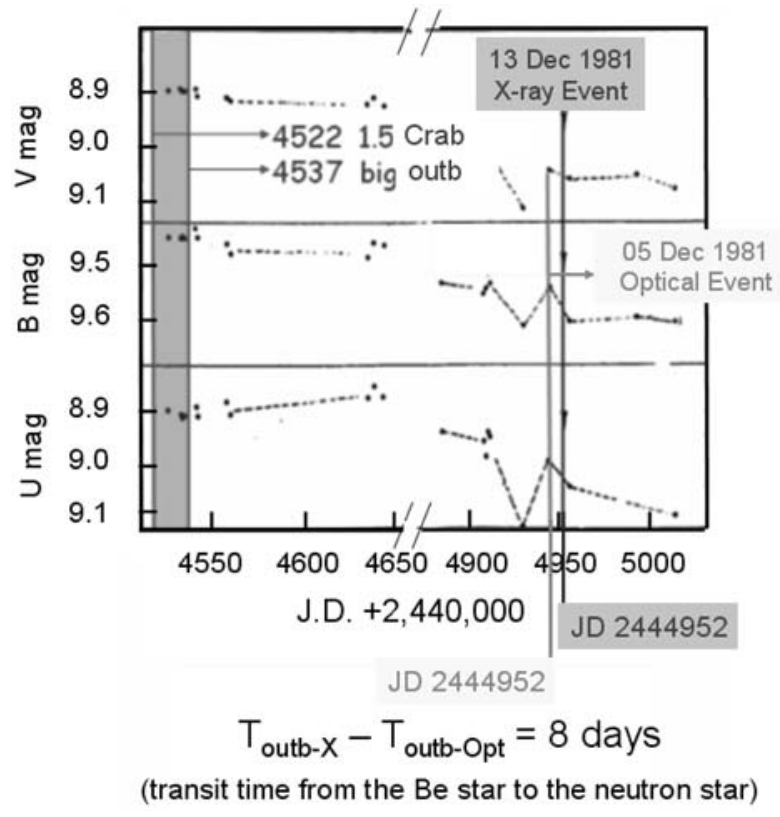

Fig. 6: Optical outburst on December 5, 1981 (811205-E) (Guarnieri et al., 1985b) - marked with a red line - occurred 8 days before the X-ray outburst of December 13, 1981 (Nagase et al., 1982) — marked with a blue line and was just 13 cycles after the 771220-E. Around the big X-ray outburst - October 9-24, 1980: JD 2,444,522 $2,444,537$ - (marked with the blue rectangle) scarce optical measurements are available

Figure 7 shows 1983-1986 X-ray outbursts of A0535+26. The long 1983 October 1-18 [(JD $(5609-5626)+2,440,000]$ X-ray outburst started just in the 6 th orbital period after the 811205 -E (or the 19 th cycle after $771220-\mathrm{E})$. No clear optical measurements were available. However, looking at the $\mathrm{V}$ light curve (obtained with the observations at the $1.5 \mathrm{~m}$ Loiano telescope), it is possible to note a small decay followed by a further increase, better detected by the Byurakan telescope (see Fig. 8) this means that the O9.7 IIIe star was experiencing a stronger mass loss process than the usual process - which could be responsible for the prolongation of the X-ray outburst. The 1984 February 1-4 [JD (5732-5735) + 2,440,000] X-ray outburst (measured with the ASTRON satellite by Giovannelli et al., 1984b; 1986a,b), probably started a few days earlier. Indeed the two available measurements (February 1 and 4) clearly show a decay. The 20th cycle after the $771220 \mathrm{E}$ is placed just at JD 5720. At this moment the $\mathrm{V}$ magnitude of the optical companion was just in a phase of decay. The following 1986 October 28-November 1 X-ray outburst, detected only twice on those dates, occurred again after the 29th cycle (JD 2,446,718) from the 771220E. Also in this case, the $\mathrm{V}$ magnitude of the optical companion was in a decay phase after a relative maximum. These three latter events strongly support that the real orbital period of the system is 110.856 days, which scans the periastron passage of the neutron star, and the X-ray outbursts are triggered with a delay of $\sim 8$ days - the transit time of the expelled material from the primary for reaching the secondary. In the case of Be star in "quiescence (steady stellar wind)", the X-ray outburst has an intensity $\approx 0.1-0.5$ Crab (normal outbursts); in the case of "moderate activity (steady stellar wind plus puffs of material expelled)", the intensity of the X-ray outburst is $\approx 0.5-1$ Crab (anomalous or noisy outbursts); in the case of the "expulsion of a shell" from the primary, preferably triggered near the periastron passage, a very strong X-ray outburst $(>1-\sim 4$ Crab) occurs and its duration is typically very long $(\approx 10-20$ days $)$ (casual outbursts).

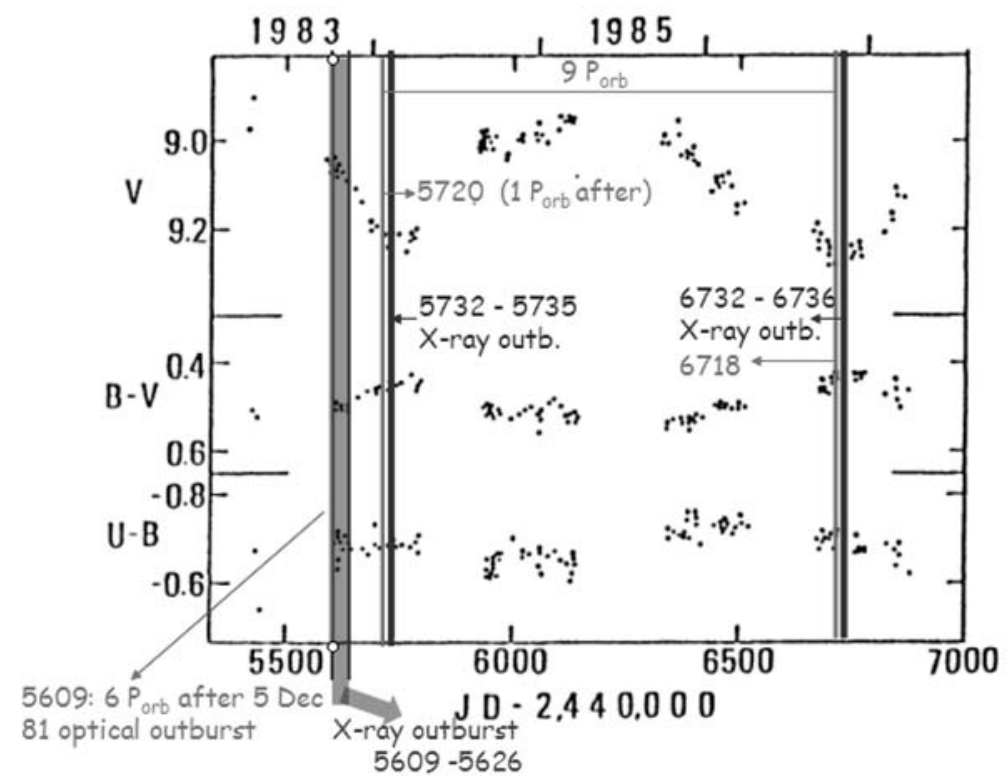

Fig. 7: 1983-1986 X-ray outbursts of A0535+26. The red lines indicate the 6th, 7th, and 16th optical cycles after the 811205-E. The blue rectangles and the blue lines indicate X-ray outbursts 


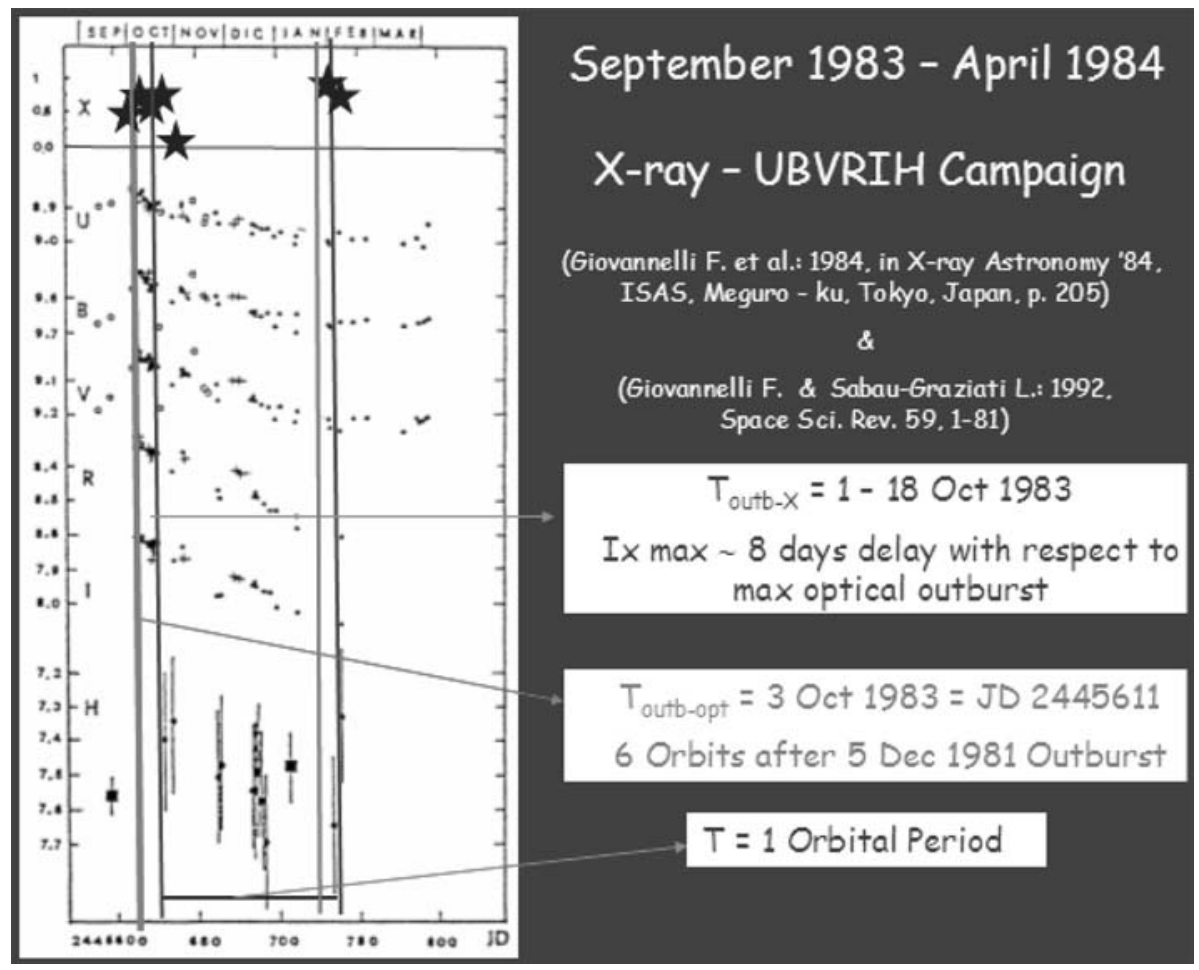

Fig. 8: UBVRI observational campaign of Flavia' star and X-ray observations with ASTRON satellite between September 1983 and April 1984 (Giovannelli et al., 1984b). Blue stars represent X-ray measurements during two outbursts (Giovannelli \& Sabau-Graziati, 1992 and references therein). Red lines indicate the 19th and 20th cycles after 771220-E

Figure 8 shows the UBVRI observational campaign of Flavia' star at the Stenberg, Loiano, Byurakan and Tirgo telescopes and X-ray observations with the ASTRON satellite between September 1983 and April 1984 (Giovannelli et al., 1984b). The blue stars represent X-ray measurements during two outbursts (Giovannelli \& Sabau-Graziati, 1992 and references therein). The optical outburst occurred on October 3, 1983 (JD 2,445,611) - practically in the 19 th cycle after the $771220-\mathrm{E}$ (or the 6 th cycle after $811205-\mathrm{E})$ - just 8 days before the X-ray maximum (JD 2,445,619). The successive X-ray outburst occurred just one cycle later [JD (5732-5735) $+2,440,000]$ with the probable maximum just at JD 5726 ; the 20th optical cycle after the 771220-E (or the 7 th cycle after 811205-E) was at JD 5720 (also visible in Fig. 7).

Figure 9 shows X-ray outbursts of A0535+26 in different epochs. Red lines mark the time of the optical cycles after 811205 -E. The X-ray activity starts roughly 8-12 days after the periastron passage of the neutron star. The strong X-ray outbursts, named casual, also occur preferably close to the periastron.

Finally, Fig. 10 shows strong optical activity of Flavia' star detected at the Loiano $1.5 \mathrm{~m}$ telescope through $\mathrm{H}_{\alpha}, \mathrm{H}_{\beta}$ and $\mathrm{H}_{\gamma}$ and $\mathrm{He} \mathrm{I}$ lines (Giovannelli et al., 2010). Such activity predicted the incoming Xray outburst of A0535+26 (Caballero et al., 2010c), as shown in Fig. 11.
Figure 11 shows the March-April 2010 X-ray outbursts of $\mathrm{A} 0535+26$. The X-ray activity starts roughly 8 days after the 93 rd cycle from the $811205-\mathrm{E}$ (Caballero et al., 2010a). The strong optical activity of Flavia' star (Giovannelli et al., 2010) was detected $\sim 10$ days before the maximum of the X-ray outburst (Caballero et al., 2010c).

\section{Discussion and conclusions}

We think we have clearly demonstrated that the optical orbital period of the A0535+26/Flavia' star system is $\mathrm{P}_{\text {orb-opt }}=110,856 \pm 0.02$ days. With this periodicity, the system scans the neutron star passage at the periastron with impressive precision. This passage is roughly 8 days before any kind of X-ray outbursts: normal, anomalous (or noisy) and casual outbursts, with some exceptions of the latter that can also occur outside this phase. An example of this could be October 9-24, 1980 (JD $2,444,522-2,444.537)$ a casual X-ray outburst apparently occurred between the 9th (JD 2,444,500.6) and 10th (JD 2,444,611.4) cycle after the 771220-E (see Fig. 6). However, according to the available measures (Giovannelli \& Sabau-Graziati, 1992 and references therein), the X-ray outburst started 22 days after the periastron passage. However, the first X-ray measure gives an intensity of $1.1 \mathrm{Crab}$, and the maximum of this strong outburst seems to be placed on October 10 

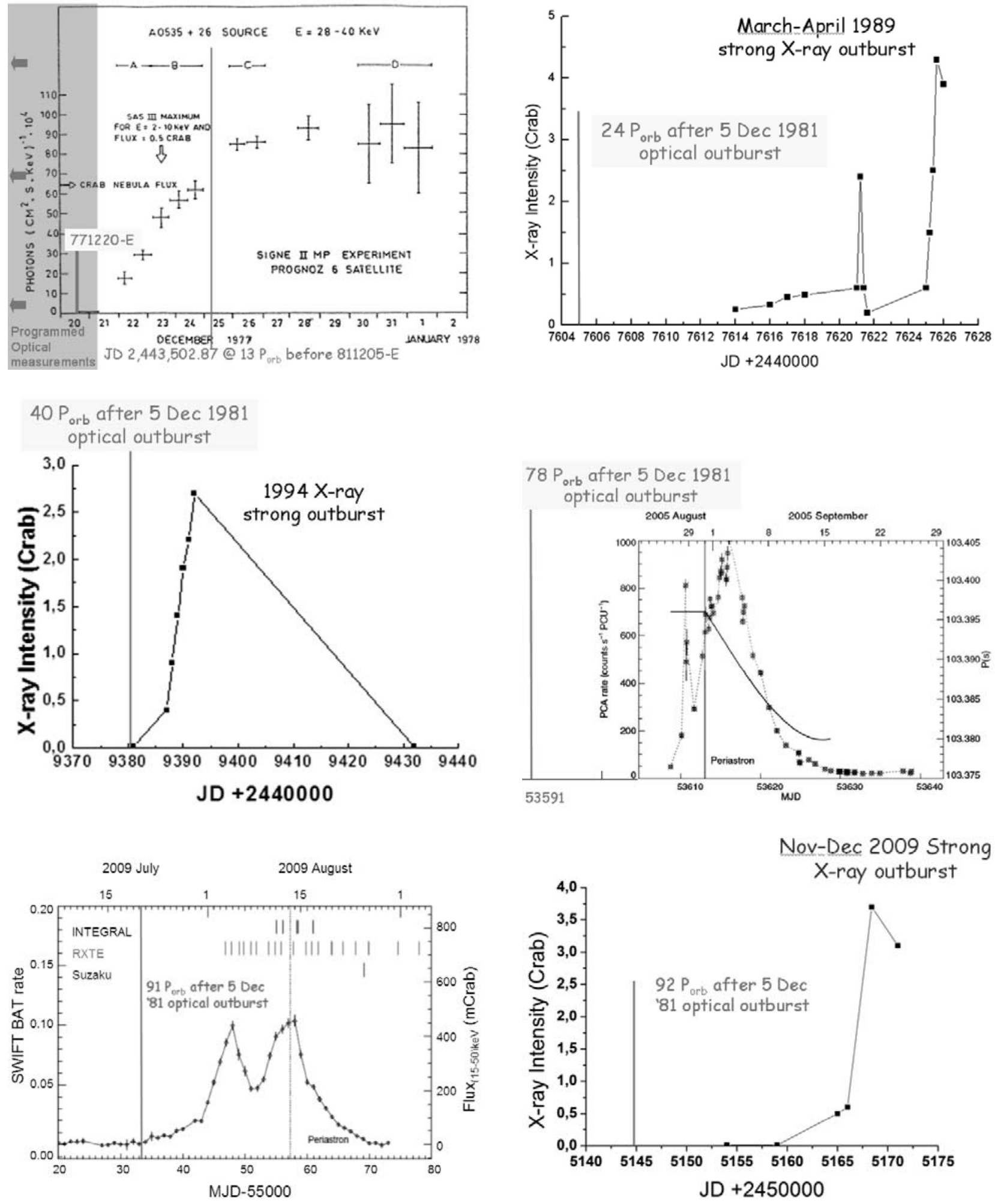

Fig. 9: X-ray outbursts of A0535+26 in different epochs. Red lines mark the time of the optical cycles after 811205-E. The X-ray activity starts roughly 8-12 days after the periastron passage of the neutron star. Also in the case of strong X-ray outbursts, named casual, they occur preferably close to the periastron. Upper left and right panels are adapted from Violes et al. (1982), and Giovannelli \& Sabau-Graziati (1992), respectively; middle left and right panels are adapted from Finger, Wilson \& Hagedon (1994), and Caballero (2009), respectively; lower left and right panels are adapted from Caballero et al. (2010b, and Caballero et al. (2010c), respectively 


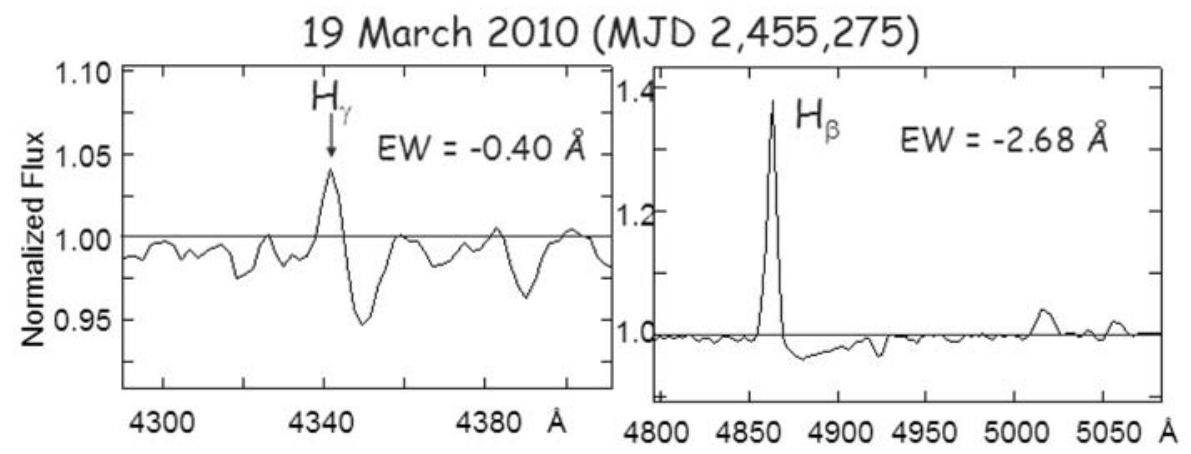

(Giovannelli, F., Gualandi, R., \& Sabau-Graziati, L.: 2010. ATel \# 2497)

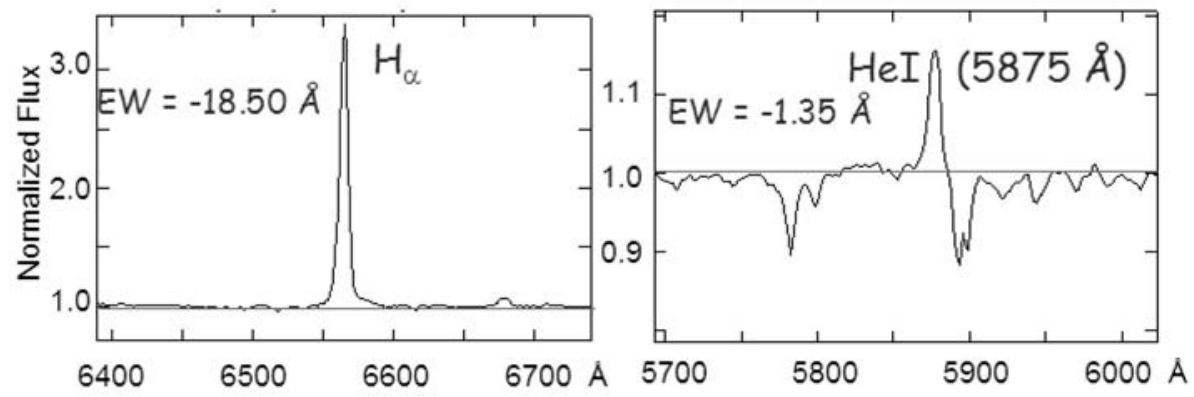

Fig. 10: Strong optical activity of Flavia' star detected at the Loiano $1.5 \mathrm{~m}$ telescope through $\mathrm{H}_{\alpha}, \mathrm{H}_{\beta}$ and $\mathrm{H}_{\gamma}$ and $\mathrm{He}_{\mathrm{I}}$ lines. The fluxes are normalized to the continuum (Giovannelli et al., 2010)

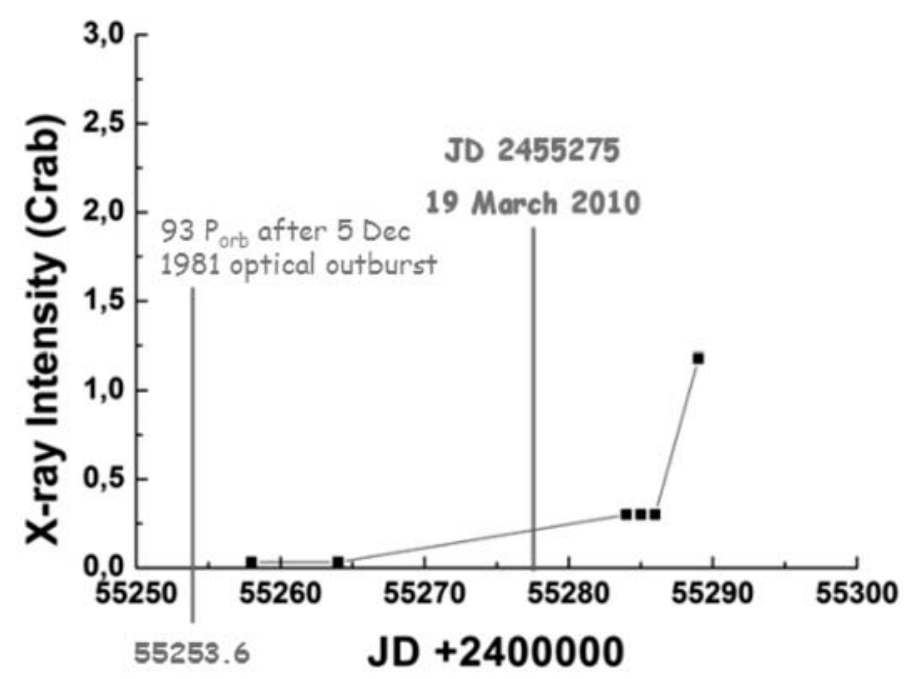

Fig. 11: March-April 2010 X-ray outbursts of A0535+26. The thin red line marks the time of the 93rd optical cycles after 811205 -E. The bold red line marks the detected strong activity of Flavia' star

(1.5 Crab). The decay of the outburst extends until October 24 (0.4 Crab) - the last measure available. Then if we extrapolate the same shape of the decay to the rise of the outburst, we could have had the same intensity as on October 24 roughly on September 27 (JD 2,444,509). This date is just about 8 days after the 9 th cycle after the 771220 -E! This could support the idea that all the outbursts are triggered around the periastron passage, even those referred to as casual (strong).

In conclusion, we have demonstrated that the optical ephemerides of the system can be given by

$$
\begin{aligned}
& \mathrm{JD}_{\text {opt-outb }}=\mathrm{JD}_{0}(2,444,944) \pm \mathrm{n}(110.856 \pm 0.02) \\
& \text { days. }
\end{aligned}
$$

Then the periastron passage of the neutron star is scanned every 110.856 days, and the X-ray outbursts are triggered starting from that moment and occur roughly after 8 days - the transit time of material expelled from the primary to reach the secondary. In the case of Be star in "quiescence (steady stellar wind)", the X-ray outburst has an intensity $\approx 0.1-0.5$ Crab (normal outbursts); in the case of "moderate activity (steady stellar wind plus puffs of material 
expelled)", the intensity of the X-ray outburst is $\approx 0.5-1 \mathrm{Crab}$ (anomalous or noisy outbursts); in the case of the "expulsion of a shell" from the primary, preferably triggered near the periastron passage, a very strong X-ray outburst $(>1-\sim 4$ Crab) occurs and its duration is typically very long $(\approx 10-20$ days $)$ (casual outbursts).

Therefore, continuous long-term monitoring of A0535+26/Flavia' star at least in optical and X-ray could definitively prove our conclusions. We believe that this behaviour of A0535+26/Flavia' star system is typical for all the X-ray/Be systems for which we wish methodical multifrequency monitoring.

In conclusion, with this paper we want to give a hint to the community to think again about the mechanisms responsible for outbursts in X-ray pulsars, and about new overall models of X-ray/Be systems.

\section{Acknowledgement}

We are pleased to thank the organizers of the Karlovy Vary 7th INTEGRAL/BART Workshop. One of us (FG) wishes to thank the LOC for logistical support.

This research has made use of NASA's Astrophysics Data System.

\section{References}

[1] Bartolini, C., Guarnieri, A., Piccioni, A., Giangrande, A., Giovannelli, F.: IAU Circ. No. 3167 , 1978.

[2] Bartolini, C., Bianco, G., Guarnieri, A., Piccioni, A., Giovannelli, F.: Hvar Obs. Bull. 7(1), 1983, 159.

[3] Bisnovatyi-Kogan, G. S. A $\&$ A, 245, 528, 1991.

[4] Burger, M., van Dessel, E., Giovannelli, F., Sabau-Graziati, L., Bartolini, C., et al.: in Multifrequency Behaviour of High Energy Cosmic Sources, F. Giovannelli \& L. Sabau-Graziati (eds.), Mem. S.A.It. 67, 365, 1996.

[5] Caballero, I.: Ph. D. Thesis, University of Tübingen, Germany, 2009.

[6] Caballero, I., Lebrun, F., Rodriguez, J., Soldi, S., Mattana, F., et al.: ATEL, No. 2496 , 2010a.

[7] Caballero, I., Kretschmar, P., Pottschmidt, K., Santangelo, A., Wilms, J., et al.: AIP Conf. Proc. $1248,147,2010$ b.

[8] Caballero, I., Santangelo, A., Pottschmidt, K., Klochkov, D., Rodriguez, J., et al.: ATEL, No. 2541, 2010c.

[9] Chartres, M., Li, F.: IAU Circ. No. 3154, 1977.
[10] Coe, M. J., Carstairs, I. R., Court, A. J., Davies, S. R., Dean, A. J., et al. MNRAS 243, 475, 1990 .

[11] Coe, M. J., Reig, P., McBride, V. A., Galache, J. L., Fabregat, J.: MNRAS, 368, 447, 2006.

[12] Corbet, R. H. D.: A\&A, 141, 91, 1984.

[13] Corbet, R. H. D.: MNRAS, 220, $1047,1986$.

[14] Finger, M. H., Wilson, R. B., Hagedon, K. S.: IAU Circ. No. 5 931, 1994.

[15] Ghosh, P.: in The Evolution of X-Ray Binaries, S. S. Holt \& C. S. Day (eds.), AIP Conf. Proc. 308, 439, 1994.

[16] Giangrande, A., Giovannelli, F., Bartolini, C., Guarnieri, A., Piccioni, A.: A \& A Suppl. Ser. 40, 289, 1980.

[17] Giovannelli, F.: The Impact of Multifrequency Observations in High Energy Astrophysics, Ph.D. Thesis, University of Barcelona, Spain, 2005.

[18] Giovannelli, F.: ChJA \& A Suppl. 8, 237, 2008.

[19] Giovannelli, F., de Loore, C., Bartolini, C., Burger, M., Ferrari-Toniolo, M., et al.: in Proc. of the Third European IUE Conference, ESA SP-176, 233, 1982.

[20] Giovannelli, F., Ferrari-Toniolo, M., Persi, P., Bartolini, C., Guarnieri, et al.: in Proc. Fourth European IUE Conference, E. Rolfe (ed.), ESA SP-218, 439, 1984a.

[21] Giovannelli, F., Ferrari-Toniolo, M., Persi, P., Golynskaya, I. M., Kurt, V. G., et al.: in $X$ Ray Astronomy '84, M. Oda and R. Giacconi (eds.), Institute of Space and Astronautical Science, Tokyo, 1984b, p. 205.

[22] Giovannelli, F., Ferrari-Toniolo, M., Persi, P., Golynskaya, I. M., Kurt, V. G., et al.: in Multifrequency Behaviour of Galactic Accreting Sources, F. Giovannelli (ed.), Edizioni Scientifiche SIDEREA, Roma, 1985, p. 284.

[23] Giovannelli, F., Kurt, V. G., Sheffer, E. K.: IAU Circ. No. 4284,1986 a.

[24] Giovannelli, F., van Dessel, E. L., Burger, M., de Loore, C., Bartolini, C., et al.: in New Insights in Astrophysics. Eight Years of UV Astronomy with IUE, ESA-SP 263, 459, 1986b.

[25] Giovannelli, F., Ziółkowski, J.: AcA, 40, 95, 1990. 
[26] Giovannelli, F., Sabau-Graziati, L.: $S S R, \mathbf{5 9}, 1$, 1992.

[27] Giovannelli, F., Sabau-Graziati, L.: in The Chemical Evolution of the Milky Way: Stars versus Clusters, F. Matteucci \& F. Giovannelli (eds.), Kluwer Publ. Co., ASSL 255, 151, 2000a.

[28] Giovannelli, F., Sabau-Graziati, L.: $A p \& S S$, 276, 67, 2001.

[29] Giovannelli, F., Sabau-Graziati, L.: $S S R, \mathbf{1 1 2}$, 1,2004 .

[30] Giovannelli, F., Bernabei, S., Rossi, C., SabauGraziati, L.: $A \& \&$ A, 475, 651, 2007.

[31] Giovannelli, F., Gualandi, R., Sabau-Graziati, L.: ATEL, No. 2497, 2010.

[32] Grove, J. E., Strickman, M. S., Johnson, W. N., Kurfess, J. D., Kinzer, R. L., et al.: ApJL, 438, L25, 1995.

[33] Guarnieri, A., Bartolini, C., Piccioni, A., Giovannelli, F.: in Multifrequency Behaviour of Galactic Accreting Sources, F. Giovannelli (ed.), Edizioni Scientifiche SIDEREA, Roma, p. 318, 1985a.

[34] Guarnieri, A., Bartolini, C., Piccioni, A., Giovannelli, F.: in Multifrequency Behaviour of Galactic Accreting Sources, F. Giovannelli (ed.), Edizioni Scientifiche SIDEREA, Roma, p. 310, 1985b.

[35] Johnston, R.: 2005. http://www.johnstonsarchive.net/relativity/ binpulstable.html

[36] Li, X.-D., van den Heuvel, E. P. J.: $A \& A L$, 314, L13, 1996.

[37] de Loore, C., Giovannelli, F., van Dessel, E. L., Bartolini, C., Burger, M., et al.: $A \& \&$, 141, 279, 1984.

[38] Margon, B., Nelson, J., Chanan, G., Bowyer, S., Thorstensen, J. R.: ApJ, 216, 811, 1977.

[39] de Martino, D., Waters, L. B. M. F., Giovannelli, F., Persi, P.: in Two-Topics in X-Ray Astronomy, E. Rolfe (ed.), ESA, SP-296, 519, 1990.
[40] Maslennikov, K. L.: Sov. Astron. Lett. 12, 191, 1986.

[41] Motch, C., Stella, L., Janot-Pacheco, E., Mouchet, M.: ApJ, 369, 490, 1991.

[42] Nagase, F., Hayakawa, S., Kunieda, H., Makino, F., Masai, K., et al.: ApJ, 263, 814, 1982.

[43] van Oijen, J. G. J.: $A \mathscr{G} A, \mathbf{2 1 7}, 115,1989$.

[44] Piccioni, A., Bartolini, C., Bernabei, S., Galleti, S., Giovannelli, F., Guarnieri, A., SabauGraziati, L., Valentini, G., Villada, M.: in The Be Phenomenon in Early-Type Stars, IAU Colloquium 175, Myron A. Smith \& Huib F. Henrichs (eds.), ASP Conf. Proc. Vol. 214, 569, 2000 .

[45] Priedhorsky, W. C., Terrell, J.: Nature, 303, 681, 1983.

[46] Rajoelimanana, A. F., Charles, P. A., Udalski, A.: 2010, arXiv 1012.4610.

[47] Rappaport, S., Joss, P. C.: in X-Ray Astronomy with the Einstein Satellite, R. Giacconi (ed.), D. Reidel Publ. Co., Dordrecht, Holland, 1981, p. 123.

[48] Rosenberg, F. D., Eyles, C. J., Skinner, C. G., Willmore, A. P.: Nature, 256, 631, 1975.

[49] Skinner, G. K.: Nature, 297, 568, 1982.

[50] Violes, F., Niel, M., Bui-van, A., Vedrenne, G., Chambon, G., et al.: ApJ, 263, 320, 1982.

Franco Giovannelli

E-mail: franco.giovannelli@iasf-roma.inaf.it

INAF - Istituto di Astrofisica Spaziale e Fisica Cosmica - Roma,

Area di Ricerca di Roma-2

Via del Fosso del Cavaliere 100, I 00133 Roma, Italy

Lola Sabau-Graziati

INTA - Dpt de Programas Espaciales y Ciencias del Espacio

Ctra de Ajalvir Km 4 - E 28850

Torrejón de Ardóz, Spain 